\title{
FORECASTING EFFECTS OF MISO ACTIONS: AN ABM METHODOLOGY
}

\author{
Chris Weimer \\ J.O. Miller \\ Mark Friend \\ Department of Operational Sciences \\ Graduate School of Engineering and Management \\ Air Force Institute of Technology \\ Wright Patterson AFB,OH 45433 USA
}

\author{
Janet Miller \\ Sensors Directorate \\ Air Force Research Laboratory \\ 2241 Avionics Way \\ Wright Patterson AFB, OH 45433, USA
}

\begin{abstract}
Agent-based models (ABM) have been used successfully in the field of generative social science to discover parsimonious sets of factors that generate social behavior. This methodology provides an avenue to explore the spread of anti-government sentiment in populations and to compare the effects of potential Military Information Support Operations (MISO) actions. We develop an ABM to investigate factors that affect the growth of rebel uprisings in a notional population. Our ABM expands the civil violence model developed by Epstein by enabling communication between agents through a genetic algorithm and by adding the ability of agents to form friendships based on shared beliefs. We examine the distribution of opinion and size of sub-populations of rebel and imprisoned civilians, and compare two counterpropaganda strategies. Analysis identifies several factors with effects that can explain some real-world observations, and provides a methodology for MISO operators to compare the effectiveness of potential actions.
\end{abstract}

\section{INTRODUCTION}

In the last decade, the United States has found itself fighting wars on a battle space it has little expertise with: the hearts and minds of populations whose support can make or break a campaign. This sort of campaign relies heavily upon Military Information Support Operations (MISO), operations whose purpose is "to induce, influence, or reinforce the perceptions, attitudes, reasoning, and behavior of individuals, foreign leaders, groups, and organizations in a manner advantageous to US forces and objectives" (Department of the Air Force 2011, p. 2).

MISO is a difficult task. The effects are nearly impossible to measure due to confounding nuisance factors outside of the operators' control, and experimentation is not ethically viable. Therefore, forecasting of effects has traditionally relied upon subject matter experts armed with sophisticated intelligence products (Department of the Air Force 2005). This research develops an agent-based model (ABM) of civil rebellion in a generalized population and allows experimentation using MISO agents to compare effects of different strategies.

This paper begins with a brief background on social simulation with a focus on ABM. We also present some recent computational social science efforts related to our MISO application. The following section provides a detailed overview of our simulation based on Epstein's civil violence model (Epstein 2006). A hypothetical application scenario is then presented, with comparison of options that may be available to the MISO planner. Results and analysis are discussed as well as a broad range of potential avenues for future research. 
Weimer, Miller, Friend, and Miller

\section{BACKGROUND}

ABM of sociological phenomena is not new; one of the first ABMs examined racial segregation in housing (Schelling 1971). Advances in computer processing have enabled greater use of this technique in the last two decades. Epstein and Axtell's (1996) Sugarscape marked the beginning of a research paradigm termed Generative Social Science (GSS). The key desideratum of GSS is the use of the simplest possible set of rules to explain an emergent behavior of interest (Epstein 2006). GSS has gained popularity as a methodology, and examples of its application can be found in many of the social sciences including economics (Zhang et al. 2010, Roozmand et al. 2011) archaeology (Epstein 2006), and sociology (Gorman et al. 2006; Mas, Flache, and Helbing 2010). In psychology, Epstein (2006) generated thoughtless application of norms in an ABM and Willer, Macy, and Kuwabara (2009) supported this with laboratory experiments showing support of norms that disagree with personal beliefs. This demonstrates the potential for GSS and traditional experimentation to augment each other.

Looking specifically at military or MISO related applications, Wragg (2006), implements dynamic social impact theory within an ABM simulating the process of opinion change for polio vaccination in Uttar Pradesh, India. His analysis combines word-of-mouth and mass media broadcasting for agent communication and considers religious affiliation, religious tolerance, and volatility for individual agent characteristics. His study shows the potential of using an ABM to measure the impact of information operations (MISO) on population opinion or behavior (Wragg 2006). In a report to the Defense Threat Reduction Agency under the Threat Anticipation Program, a group of authors from Los Alamos National Laboratory discuss their research using computational social simulation in understanding Islamist political violence (Watkins et al. 2008). They demonstrate their approach using both a systems dynamic model and an ABM, highlighting the difficulty in finding not only commonly accepted social science theory but also valid mathematical models to capture required social science phenomena within a simulation. Epstein's GSS approach (2006), to social science is highlighted as an accepted method to model macrobehavior based on micro-level rules (Watkins et al. 2008). Other recent work includes a number of efforts sponsored under the Office of the Secretary of Defense (OSD) Human Social Culture Behavior Modeling (HSCB) Program (Boiney and Klein 2011). The technical investment area under this program most closely associated with our research is computational modeling. Also worth mentioning in this brief discussion of military related research and tools is the tactical conflict assessment and planning framework (TCAPF) developed by the US Agency for International Development (USAID) (2010) and also contained in Army Handbook 10-14, Assessment and Measures of Effectiveness in Stability Operations (U.S. Army 2010). The purpose of the TCAPF is to provide a method and the tools to collect culturally sensitive and consistent data, identify local causes of instability, develop programs to reduce instability, and measure the effectiveness of the programs (Watkins et al. 2008). The TCAPF could use some type of MISO as a program and ties directly into computational modeling in terms of providing input data as well as a snapshot of actual behavioral changes that we might desire to capture in a simulation.

Epstein's civil violence model (2006) serves as the basis for our ABM. As presented in detail in Weimer (2012), we expand on Epstein's work to add communication between civilians and movement that is more grounded in influence psychology, specifically the concept of liking as presented by Cialdini (2007).

\section{CIVIL REBELLION SIMULATION}

In order to be generalizable across situations, the social environment cannot be modeled after any individual nation or culture. Rather, fields are provided that can be manipulated to better reflect a given culture. In order to facilitate comparisons to previous work by Epstein (2006) and to aid in verification and validation of simulation output, we adhere closely to Epstein's model and maintain every qualitative trait 
observed in his analysis. Additional inputs used are values that produced an average response in previous research by Weimer (2012).

Note that the strength of this abstraction is an appropriate comparison between treatments, rather than actual forecasting of specific levels of rebellion or anti-government sentiment. To accomplish the latter, every variable that affects rebellions would have to be accounted for, which would make for a very complicated and over-specified model.

All programming is done using Repast Simphony 2.0 Beta. An image of the simulation is shown in Figure 1. Two types of agents are interacting in the basic social landscape: Civilians and Cops. MISO agents are later added for experimentation. The underlying virtual space the agents move about in is a 40 cell by 40 cell torus. Each cell can hold only a single Civilian or Cop agent. All agents move in random order each simulated click of time according to agent specific behavior described in the following sections.

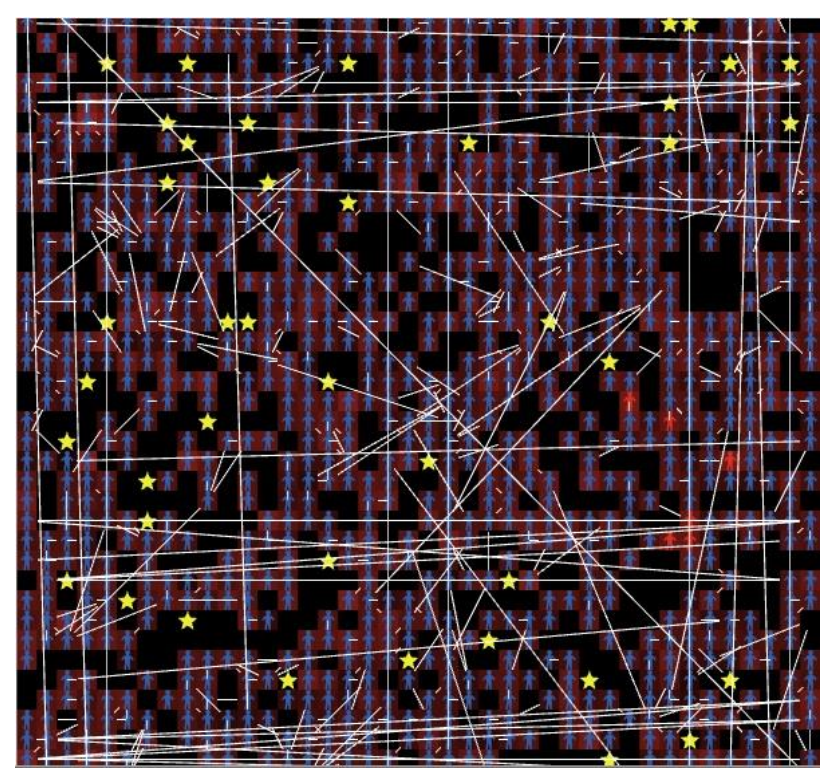

Figure 1: Screenshot of simulation portraying Civilians (people) colored according to whether they are active rebels (red) or not (blue) exhibiting grievance (background scaled black to red), friendships (arrows), and Cops (gold stars)

\subsection{Civilian Behavior}

Civilians are represented by people in the visualization, and their logic is shown in Figure 2. The level of grievance felt toward the government is represented as opinionGene in the manner of a genetic algorithm as introduced by Holland (1995). A value of 1 on the opinionGene represents an aggrieved opinion, whereas a value of 0 represents a non-aggrieved opinion. Overall grievance is therefore considered the mean value of 20 individual elements within the opinionGene, each represented by a binary digit, scaled down by the legitimacy of the government, which is static in this analysis at 0.82 . That is

$$
\text { Grievance }=\left(\frac{1}{20} \sum_{i=1}^{20} \text { OpionionGene }_{i}\right)(1-\text { Legitimacy })_{\text {. }}
$$


Weimer, Miller, Friend, and Miller

For ease of presentation, we refer instead to grievance as

$$
\text { Grievance }^{\prime}=\sum_{i=1}^{20} \text { OpinionGene }_{i}
$$

After a Civilian moves to a randomly chosen empty cell within its movement range, it examines its surroundings and decides whether it should become actively rebellious. To do so, it counts both the number of Cops $(C)$ and the number of active rebels $(A)$ in its vision range (civVision), and computes an estimated probability of arrest $(P)$ (Epstein 2006),

$$
P=1-e^{-2.3\left(\frac{C}{A}\right)_{c i v i s i o n}}
$$

The Civilian agent then calculates net risk $(N)$ by multiplying this probability by its risk tolerance $(R)$, $N=R P$. If the difference between grievance and $N$ exceeds a threshold (rebelThreshold), set here to 0.1, the Civilian will become an active rebel. Otherwise, it will remain inactive. As an active rebel a Civilian can be arrested by a Cop for a random time between 1 and 30 steps. Arrested Civilians cannot be seen and do not occupy a cell during their time in jail. After its jail term the Civilian returns to a randomly selected open cell.

After choosing a state, a Civilian will randomly choose a target Civilian from its Moore neighborhood, the eight bordering cells, with whom to communicate. A random topic, or index of the opinion gene, is chosen to discuss, and if the two Civilians' opinions differ, the target Civilian will change their opinion. If the 1-norm distance between the Civilians' opinion genes is less than $25 \%$ of the possible difference, a friendship will be formed, and for the next 20 ticks the two Civilians will prefer to move toward each other. There is also a $1 \%$ chance of a mutation, the alteration of a random opinion within the source's opinion gene. This prohibits opinions from going extinct over time.

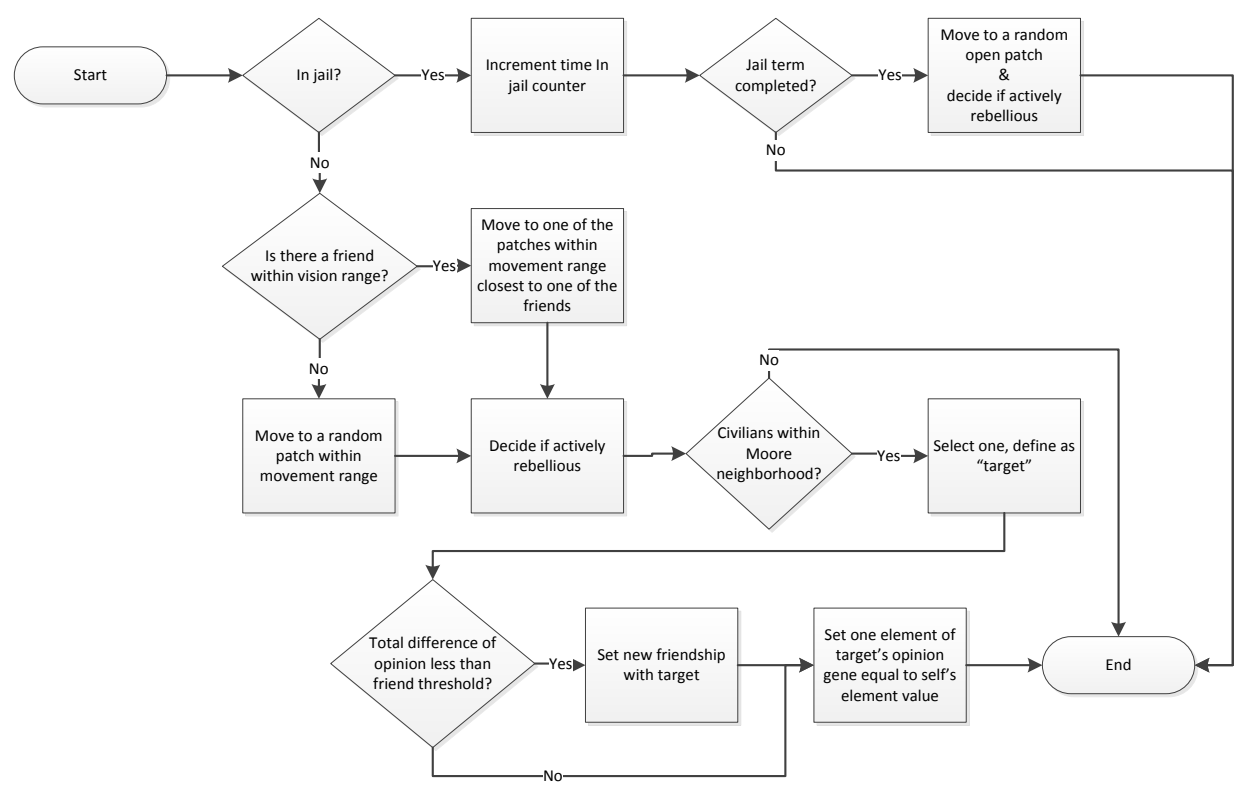

Figure 2: Civilian logic flow 


\subsection{Cop Behavior}

Cops are far simpler than Civilians, as shown by their logic flow in Figure 3. Before moving, a Cop examines the cells within its vision looking for active rebels. If it sees any, it moves to one of their locations and arrests that rebel for a random period of time between 1 and 30 steps. If there are no rebels within the Cop's vision, it will randomly move to an empty block within its vision.

Figure 3: Cop logic flow

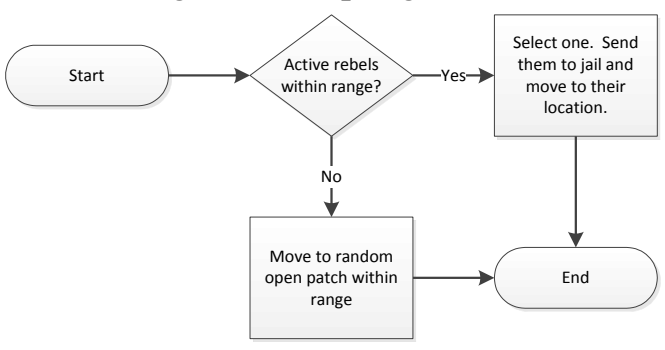

\subsection{MISO Agents}

MISO agents are those added at a random location to the base simulation as described above for the purpose of experimentation. Here we have coded an agent whose behavior can be modified to act in many roles by modifying variable values. MISO agents' effectiveness is a function of their affiliation (government or rebel), government legitimacy, their media (written or internet), range of influence (commRange), the number of opinions about which they communicate (commBreadth), and the number of contacts that can be made in a turn (commAttempts). Two forms of this agent are used in this case study: a pamphlet distributor and an internet campaigner. The values associated with each are shown in Table 1.

Table 1. Variable values for two types of MISO agents

\begin{tabular}{|c|c|c|}
\hline Variable & Pamphlet Distributor & Internet Campaigner \\
\hline Affiliation & Government/Rebel & Government/Rebel \\
\hline Susceptible Population & Literate Civilians & Web-connected Civilians \\
\hline commRange & 3 & 40 \\
\hline commBreadth & {$[1,20]$} & {$[1,20]$} \\
\hline commAttempts & 10 & 10 \\
\hline
\end{tabular}

Every turn, the MISO agent chooses a target list of size commAttempts within range commRange from those susceptible to its influence. For each target on this list, one of the commBreadth topics to which they are assigned is chosen, and the target's opinion on that topic is set, if rebel, to one with probability ( 1 - legitimacy), or if government, to zero with probability (legitimacy). Agents with written messages may only affect literate Civilians, and agents with internet messages may only affect web-connected Civilians. Generally, internet range is also unlimited, which is modeled using commRange $=40$ rather than the pamphlet range of commRange $=3$. 


\section{APPLICATION AND ANALYSIS}

In this analysis, we pose a hypothetical scenario in which a population within an area we are interested in is being affected by a rebel pamphlet propaganda campaign. In this hypothetical case, the area of interest has been modeled in the past, and the model settings in Table 2 produced appropriate responses and therefore are assumed as ground truth. Literacy and internet connectivity rates for the global average are used and taken from the CIA World Factbook (2012), but country-specific values could be found in the same manner. The rebel propaganda campaign is reported to have a moderate level of focus, equivalent to $25 \%$ of possible anti-government topics. Thus, commBreadth is set to 5 for the rebel agent.

Table 2: Values used in simulation for application scenario

\begin{tabular}{|c|c|}
\hline Variable Name & Value \\
\hline civVision & 4 \\
\hline civRange & 4 \\
\hline copVision & 4 \\
\hline popDensity & 0.5 \\
\hline copDensity & 0.04 \\
\hline legitimacy & 0.82 \\
\hline literacy & 0.84 \\
\hline connectivity & 0.30 \\
\hline
\end{tabular}

Due to budget and political constraints, only one counter-rebel campaign may be implemented. We consider either a pamphlet campaign or an internet campaign with pro-government information. The determination of message focus is left to the MISO planner. The goal of the propaganda campaign is to minimize Civilians' mean grievance.

The purpose of this experiment is to demonstrate the program's potential utility for MISO campaign planning. There would almost certainly be changes to the grievance response if legitimacy, literacy, and connectivity were changed, but we assume for the purposes of this experiment that these factors are fixed.

\subsection{Information Medium}

To determine the optimal medium for information, an experimental design was implemented examining the four conditions of interest: no action, pamphlet campaign, internet campaign, and both campaigns. Each design point consisted of 5 replications with a 500 tick run length. All MISO agents for this analysis used commBreadth of 5, which is equivalent to the rebel pamphleteer. While the use of both campaigns has been determined not to be a choice due to budget constraints, it may be interesting for the decisionmaker to see the estimated combined effect of the campaigns. A plot of the mean grievance shown in Figure 4 demonstrates the best choice of campaign is dependent upon the campaign length. If the goal has a short-term focus, the pamphlet campaign serves as the most effective response to the rebel message; if the focus is more long-term, the internet campaign serves as the most effective. The cumulative effect of introducing both campaigns is certainly stronger than either campaign alone. As shown in Figure 5, this translates to decreased rebellious activity, though the higher noise in this variable obscures the shortterm difference between pamphlet and internet responses. 


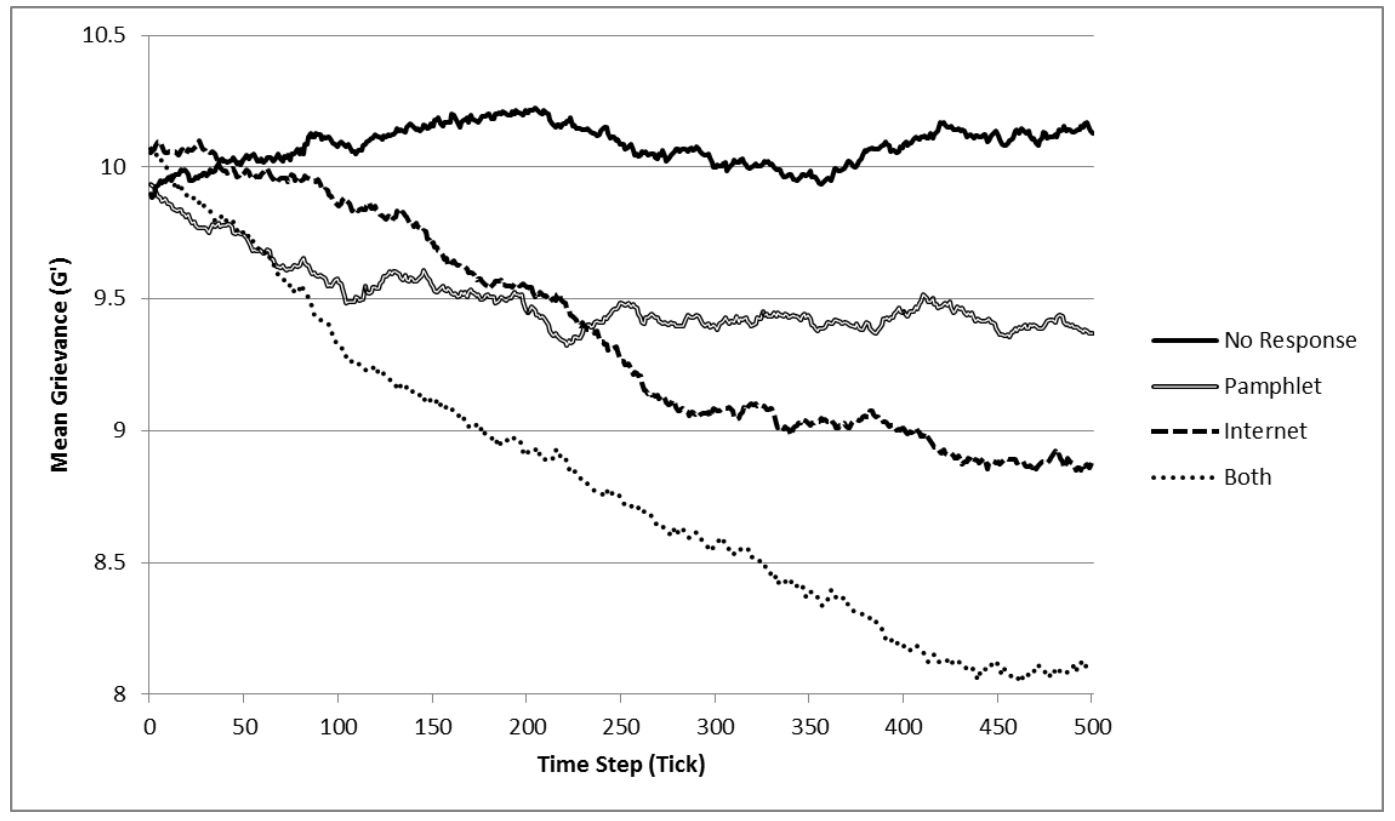

Figure 4: Civilian grievance response to pro-government information campaigns

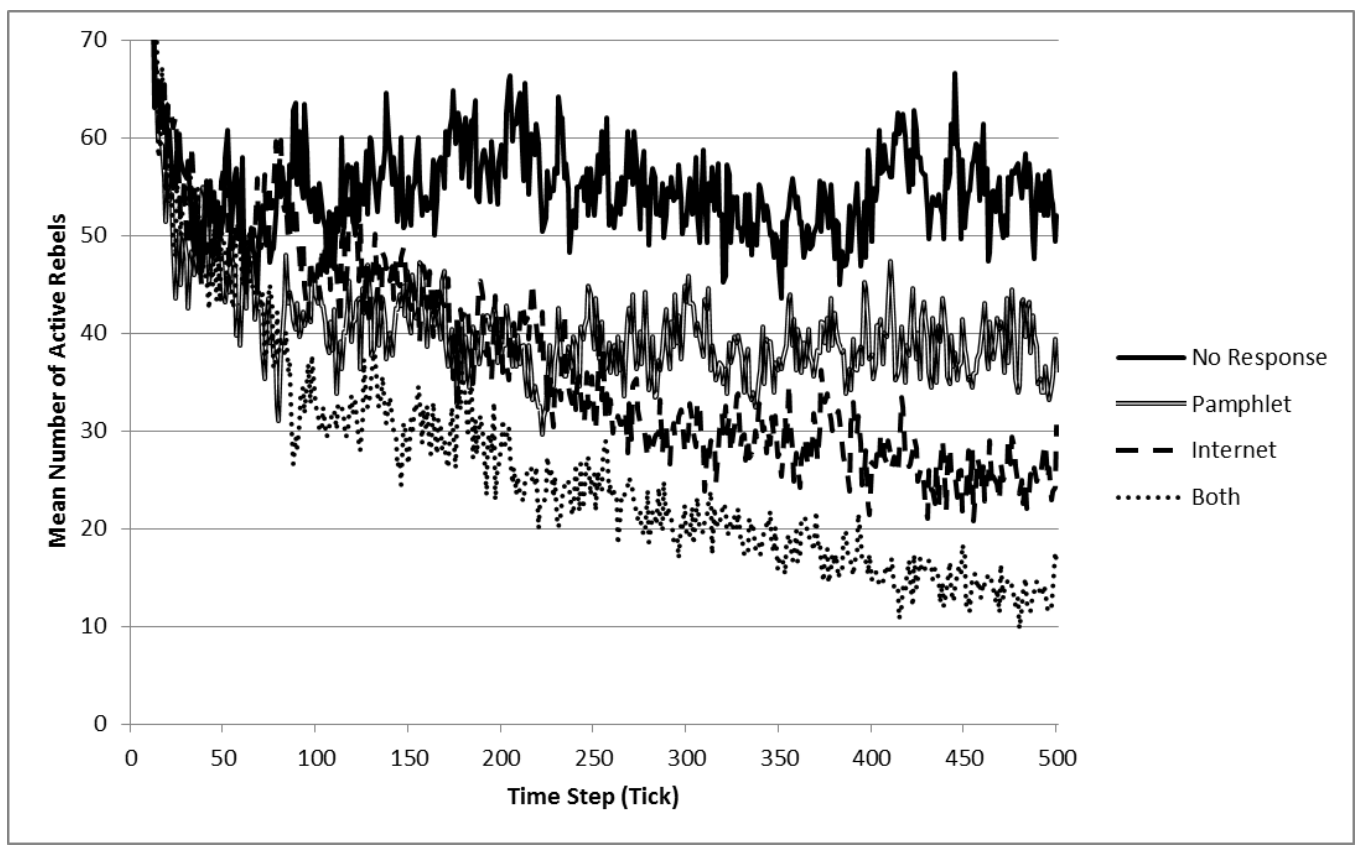

Figure 5: Civilian rebellion response to pro-government information campaign

\subsection{Topical Focus}

Because neither medium was ruled out in the first experiment, we performed another experiment for both pamphlet and internet campaigns. We expected significant curvature in the effect of message breadth, so we performed 2 runs at each level of breadth (every integer in $[1,20]$ ) for each medium, for a total of 80 replications. The effect is not statistically significant early in a run. At ticks 100, 200, 300, and 400 
where the difference between internet and pamphlet responses was greatest, there is no evidence of breadth affecting grievance.

At tick 500, there is strong evidence $(p<0.0001)$ of a negative linear effect of breadth upon grievance. There is insufficient evidence to show that this effect differs between treatments. Breadth and campaign type explain $44.4 \%$ of variance in grievance. The majority of observed variance, then, is attributable simply to noise, as nothing else is altered between runs. The associated ANOVA is shown in Table 3.

Table 3. ANOVA for Breadth Effect on Grievance

\begin{tabular}{|l|c|c|c|c|c|}
\hline \multicolumn{1}{|c|}{ Source } & DF & Sum of Squares & Mean Square & F Ratio & p-value \\
\hline Model & 2 & 9.031941 & 4.51597 & 30.73 & $<.0001$ \\
\hline Type & 1 & 2.616809 & 2.616809 & 7.8067 & $<.0001$ \\
\hline Breadth & 1 & 6.415132 & 6.415132 & 43.6533 & $<.0001$ \\
\hline Error & 77 & 11.31564 & 0.14696 & & \\
\hline Lack Of Fit & 37 & 6.071054 & 0.164083 & 1.2514 & 0.2434 \\
\hline Pure Error & 40 & 5.244583 & 0.131115 & & \\
\hline Total & 79 & 20.34758 & & & \\
\hline
\end{tabular}

\subsection{Recommendations}

Based on the analysis of our selected hypothetical scenario and parameter settings used, we would recommend to the decision-maker to use a broad-themed internet campaign for long-term effect on civilian support for the government. For a short-term effect, breadth is inconsequential, but we would recommend a pamphlet campaign.

\section{CONCLUSIONS}

The intent of this paper is to demonstrate the flexibility of ABMs and genetic algorithms to compare MISO actions in a computational social simulation. Using simple yet powerful scenarios we have shown that this approach provides insights useful for MISO campaign planning. This serves as a proof of concept, and additional research must be done to validate the model before implementing it in this way. As we had stated previously, this abstraction is not designed to capture a specific real-world scenario or an attempt to forecast specific levels of rebellion or anti-government sentiment. However, the structure of the model is designed to allow use of real-world data in defining parameters for analysis including individual elements for the opinionGene. Real-world effects are more complicated and difficult or impossible to measure, however this technique offers insight into subtle effects that are otherwise hidden. Furthermore, as the analyst begins to better understand the effects of different variables, the number of runs, and therefore analyst time, required for proper analysis may decrease. Case in point: the curvature expected in the effect of message breadth was not found. Far less data could have been collected to analyze the effect of breadth.

Much future research can be considered. Although no formal validation was conducted, our model exhibited similar emergent social behaviors found in previous accepted studies, a reasonable measure of face validity for such a simulation (Wragg 2006). It would be interesting to attempt validating for a certain area of interest perhaps using data gathered and MISO programs evaluated using the TCAPF (United States Agency for International Development 2010, U.S. Army 2010). Even altering only literacy and web-connectivity to match a particular region would be illuminating. Our modeling approach could also serve to provide testable hypotheses for more traditional psychology and sociology research. 
Weimer, Miller, Friend, and Miller

\section{ACKNOWLEDGMENTS}

The views expressed in this article are those of the authors and do not reflect the official policy of the United States Air Force, the Department of Defense, of the US Government.

\section{REFERENCES}

Boiney, J. and G.L. Klein. 2011. "HSCB Modeling Program Overview.” HSCB Newsletter Winter 2011.

Central Intelligence Agency, 2012. "The World Factbook.” Accessed January 21, 2012. https://www.cia.gov/library/publications/the-world-factbook/

Cialdini, R.B. 2007. Influence: The Psychology of Persuasion. New York: Collins.

Department of the Air Force. 2005. "Information operations." AFDD 2-5, Washington D.C.: GPO.

Department of the Air Force. 2011. "Military information support operations (MISO)." AFI 10-702, Washington D.C.: GPO.

Epstein, J.M. 2006. Generative Social Science: Studies in Agent-Based Computation Modeling. Princeton, NJ: Princeton University Press.

Epstein, J.M., and R. Axtell. 1996. Growing Artificial Societies. Washington, D.C.: The Brookings Institution.

Gorman, D.M., J. Mezic, I. Mezic, and P.J. Gruenewald. 2006. “Agent-based Modeling of Drinking Behavior: A Preliminary Model and Potential Applications to Theory and Practice." American Journal of Public Health, 96(11), 2055-2060.

Holland, J.H. 1995. Hidden Order. New York: Basic Books.

Mäs, M., A. Flache, D. Helbing. 2010. "Individualization as Driving Force of Clustering Phenomena in Human." PLoS Computational Biology, 6(10), 1-8.

Roozmand, O., N. Ghasem-Aghaee, G.J. Hofstede, M.A. Nematbakhsh, A. Baraani, and T. Verwaart. 2011. "Agent-based Modeling of Consumer Decision Making Process Based on Power Distance and Personality." Knowledge-Based Systems, 24(7), 1075-1095.

Schelling, T.C. 1971. "Dynamic Models of Segregation.” Journal of Mathematical Sociology, 1(2), 143186.

United States Agency for International Development. 2010. Tactical Conflict Assessment and Planning Framework. Presentation with notes, USAID, Washington D.C.

United States Army. 2010. "Assessment and Measures of Effectiveness in Stability Operations." Hand book 10-41, Center for Army Lessons Learned, Ft. Leavenworth, KS.

Watkins, J.H., E.P. Mackerrow, P.G. Patelli, A.S. Eberhard, and S.G. Stradling. 2008. "Understanding Islamist Political Violence Through Computational Social Simulation." Technical Report, Defense Threat Reduction Agency, Report Number ASCO 2008-009.

Weimer, C.W., 2012. "Forecasting Effects of Influence Operations: A Generative Social Science Methodology." MS Thesis, AFIT/OR/MS/ENS/12-26, Graduate School of Engineering and Management, Air Force Institute of Technology, Wright Patterson AFB, OH.

Willer, R., M.W. Macy, and K. Kuwabara. 2009. "The False Enforcement of Unpopular Norms." American Journal of Sociology, 115(2), 451-490.

Wragg, T. 2006. "Modeling the Effects of Information Campaigns Using Agent-Based Simulation." Technical Report, Defense Science and Technology Organization, Edinburgh, Australia.

Zhang, W., G. Li, X. Xiong, Y.J. and Zhang. 2010. "Trader Species with Different Decision Strategies and Price Dynamics in Financial Markets: An Agent-based Modeling Perspective." International Journal of Information Technology \& Decision Making, 9(2), 327-344. 
Weimer, Miller, Friend, and Miller

\section{AUTHOR BIOGRAPHIES}

CHRIS WEIMER received his B.S. degree from the U.S. Air Force Academy in 2006 and M.S. degree in Operations Research from the Air Force Institute of Technology in 2012. He currently serves as Captain in the US Air Force stationed at Kirtland AFB, NM, where he is an analyst at the Air Force Operational Test and Evaluation Center (AFOTEC). His research interests include social science modeling, computer simulation, and social psychology. He can be reached at christopher.weimer@us.af.mil.

J. O. MILLER is a 1980 graduate of the U.S. Air Force Academy (USAFA) and retired from the Air Force as a Lt. Colonel in January 2003. In addition to his undergraduate degree from USAFA, he received an MBA from the University of Missouri at Columbia in 1983, his M.S. in Operations Research from the Air Force Institute of Technology (AFIT) in 1987, and his Ph.D. in Industrial Engineering from The Ohio State University in 1997. Dr. Miller is Director of the Center for Operational Analysis and an associate professor in the Department of Operational Sciences at AFIT. His research interests include combat modeling, computer simulation, and ranking and selection. He can be reached at john.miller@afit.edu.

MARK FRIEND received his B.S. degree from Texas Christian University in 1996 and M.S. and Ph.D. degrees in Operations Research from the Air Force Institute of Technology in 1998 and 2007, respectively. He currently serves as a Lieutenant Colonel in the US Air Force stationed at Wright-Patterson AFB, $\mathrm{OH}$, where he is an Assistant Professor in the Department of Operational Sciences at the Air Force Institute of Technology. His research interests lie in the areas of automatic target recognition, combat assessment simulation and applied statistics. Dr. Friend can be reached at mark.friend@wpafb.af.mil.

JANET E. MILLER received her Ph.D. in Industrial and Systems Engineering from The Ohio State University in 2002; her M.S. in Management Information Systems from The University of Arizona in 1988, her M. A. in Economics from the University of Oklahoma in 1987, and her B. S. in Electrical Engineering from Louisiana Technical University in 1984. In February 2002, she joined the Air Force Research Laboratory as a Senior Electronics Engineer and is currently in the Sensors Directorate. Her research interests include trust in the digital domain, human-systems integration, macrocognition, and visualization for complex, analytic tasks. Dr. Miller can be reached at janetoptimist@aol.com. 